\title{
A Corpus-based Study on the Influence of Chinese Subjective Thought Patterns on English Learning
}

\author{
Liwei Sun \\ School of Arts and Science, Jilin Agricultural Science and Technology College, Jilin 132101
}

Keywords: Corpus-based, Influence, Subjective Thought Pattern, English learning.

\begin{abstract}
This paper tends to discuss the influence of the Chinese subjective thought pattern on English learning. The corpus of Chinese English learners and native English speakers are employed as the source of data collection for the contrastive analysis of the influence of Chinese subjective thought patterns on English learners. According to the analysis, two findings could be concluded, firstly, Chinese English learners tend to have a preference for the use of active voice and secondly, they intends to apply more subjective words in their composition.
\end{abstract}

\section{Introduction}

Although the teaching and learning conditions such as the learning materials, English teachers, English communicating environment and the teaching methods have been improved a lot in recent years, the learners still find it very difficult to learn English well and it seems even harder to achieve the goal of English teaching. Students still have difficulties in their listening, speaking, reading and writing, especially in writing.

What is the key obstacles lying behind the process of English learning has become the essential issues for English teachers and learners to dig out. Therefore, This paper tends to explore the solution to the issue through the perspective of the influence of Chinese thought patterns.

If we were to look at the English compositions written by Chinese students, we should notice the prevalence of Chinglish. As this blend name suggests, Chinglish is a mixture of Chinese and English. It is English in form and Chinese in essence and accordingly neither English nor Chinese. As Joan Pinkham(1998) holds that Chinglish refers to words that are ungrammatically strung together, with often inappropriate lexis and probably only a partially comprehensible pronunciation. In other words, it is a deformed English phenomenon misused by Chinese people, and it obviously violates the general English rules. Its composition and its using scope are unstable and limited; besides, it does harm to international communication and the spread of Chinese civilization. Therefore, the reasons for the prevalence of Chinglish have been on the agenda. This paper assumes that Chinese thought patterns exert a tremendous influence on the low learning efficiency of English and on the formation of Chinglish and tends to explore Chinese subjective thought pattern on the influence of English learning particularly.

\section{Subjective Thought pattern VS Objective Thought pattern}

\section{Subjective Thought pattern}

The traditional Chinese culture plays an important part in the formation of this thought pattern. Firstly, Chinese culture and philosophy take human and human affairs as the core and regard man as the center of the cosmos and the measure of all things on the earth. In addition, it is well accepted by Chinese culture that if man recognizes himself, he recognizes the objective world and its working laws. This thinking can also be certified by the Chinese traditional philosophical schools, all of which are apt to mixing the two. Taoism holds that "all things on the earth are identical with man." According to Confucianism, "all things on the earth can be found in me." Both theories emphasize the central function of man in the cognition of universe on the basis of perceiving experience. The Confucian "Jen” includes not only all human beings but also the universe in totality. 
In this connection "Jen" is expanded to the concept of one body with the universe, the mind of man, the foundation of all goodness, the generative force of all things and the source of all production in the universe. Therefore, Subjective thought pattern is characterized by regarding human as the center of the cosmos, knowing the world from self, probing into self and maintaining that man and heaven, subject and object are inalienable. Dong Zhongshu maintained that only man could create prosperity between heaven and earth. All of the above ideas manifest the essential position of man. Thus, it is man the subject of cognition. If he conducts an action, it has to be conducted around him. For this reason, sentences in Chinese tend to be constructed around a topic and the frequent omission of subjects in Chinese grammar also reflects this thought pattern.

\section{Objective Thought pattern}

In contrast with the subjective thought pattern, the native English speakers tend to think objectively. Objective thought pattern regards object and nature as the core, and pays more attention to the observation and research of the natural object. According to the preceding part, this thought pattern also has something to do with the analytical one. The objective thought pattern regards nature as the cognitive object of human beings and something to conquer. The nature is completely incompatible with human beings. (Chad Chanson, 1992) Consequently, westerners strictly set apart subjective world from objective world. They insist on separating the subject of cognition from the object of cognition. Since the function of the subject is to know the object which is the focus of cognition. The westerners have long believed that all problems can be solved by science. People tend to prize objectivity, empirical evidence and the scientific method, which often clash with the values of subjectivity, mysticism and intuition in Chinese culture. "knowledge is power" of Francis Bacon is a reflection of human's ambition to conquer nature with knowledge.

In western thought pattern, human potentialities and creativity have been given full scope that western philosophers and scientists endeavored to explore the principle element of the objective reality. Then after carrying out some empirical observations and experiments on the object, they made their own judgments and developed some theories. Thus, they believe that human beings surmount nature and have absolute control over nature. The nature of human is to conquer nature with science and wisdom. ( $\mathrm{Li}, \mathrm{C}$. \& Thompson, 1976 )The formation of this thought pattern also contributes to its own cultural and philosophical basis. To begin with, the spirit of occidental culture is to separate subject from object and to conquer nature. As mentioned above, occidental thinking is linear and it puts emphasis on the co-existence and antithesis of duality and puts forward dichotomy, such as man and nature, subject and object, material and spirit, mortal and immortal, good and bad, right and wrong, peace and war, success and failure, etc. Based on dichotomy and antithesis, western culture puts stress on man's conquering of the world and nature. Man could exist only as far as they have the capacity to control it and the strength to reconstruct it. In English, the subject is the most important part of speech. As we all know that there are some basic sentence patterns in English, the most common of which are SV, SVC, SVO, SVO1O2 and SVOC.

\section{Corpus selection}

This thesis takes CLEC (Chinese Learner English Corpus) as the main source of data collection. Meanwhile, its contrastive English corpora: BROWN, LOBand FLOB are also employed so as to make a comparison between these two languages. Most examples listed in this thesis are collected from the sub-corpora of St3 and St4 in CLEC due to its large amount of data. With the assistance of the CLEC, sufficient manifestations of Chinglish will be presented in the thesis, which provide us with enough data to conduct the study and analysis.

Quantitative and qualitative analyses are two basic approaches to language study based on corpus approach. In corpus-based studies, quantitative analysis is frequency-based. Usually in quantitative analysis, observed linguistic features are classified, counted, and explained statistically. Some simple or complex charts or models are often constructed to show certain tendencies and indicate what occurrences are common or rare. In addition, based on the frequencies shown in the charts or models, comparisons of some linguistic features between different corpora become possible. While qualitative analysis focuses on the typical features of the data being studied. After 
quantitative analysis is made and the frequencies of the given linguistic features are shown, this analysis is often adopted to describe the commonplace, variation or distinction of the given linguistic features shown in the charts or model.

\section{The Influence of subjective thought pattern}

\section{The preference for active voice}

Subjective thought pattern regards man as the centre of the cosmos and the measure of all things. It emphasizes the harmony between man and nature. Thus, the idea of the unity of the animate things and the nature is deeply rooted in people's mind. In this thought pattern, man is the subject of cognition. Therefore, it is difficult to grasp man as an objective existence. In contrast, the native speakers tend to think objectively. It regards object and nature as the core, and pays more attention to the observation and research of the natural object. Thus, in this thought pattern, nature is the cognitive object of human beings and something to conquer. Such thought patterns of the Chinese and the native English speakers have a strong influence on the voice of Chinese and English. Therefore, The passive voice of the sentence is not well developed in Chinese as that in English. Even if the passive voice is put to use, it doesn't take a relatively fixed form like that of in English. Most sentences that convey the passive message are presented in the form of active voice. Thus, there are more indicators of the passive voice other than "Bei" in Chinese. For example:

[1] "Suo you ke cheng yi jing jie shu"

All the subjects have been finished.

[2]"Xi wang zheng fu xun su jie jue zhe yi wen ti”

It was hoped that the government could solve this problem without delay.

In English, the passive voice is explicitly and definitely expressed in form which is fixed. It is made up of "be" and a past participle and "by" is the most commonly used indicator. For example:

[1] History is made by the people.

[2] The song was composed by a student.

Influenced by such thought patterns, passive voice is supposed to be used less in the writing of Chinese students than that in the native English speakers. Therefore, the preference for active voice can be one of the manifestations of Chinglish. It can be proved by the following table which includes a list of words that have been used less frequently in CLEC.

Table 4.1 The words used less frequently in CLEC in comparison with FLOB

\begin{tabular}{|l|l|l|l|l|}
\hline words & $\begin{array}{l}\text { the frequency in } \\
\text { CLEC }\end{array}$ & percentage & $\begin{array}{l}\text { the frequency in } \\
\text { FLOB }\end{array}$ & percentage \\
\hline been & 1156 & 0.1 & 2845 & 0.23 \\
\hline by & 3443 & 0.29 & 5418 & 0.44 \\
\hline
\end{tabular}

As can be seen from the table that the indicator of passive voice such as "been" and "by" is used less in CLEC than that in FLOB. It proved that the preference for active voice or the incapability of applying passive voice is a weak point of Chinese English learners. In addition, the confusion of the students when writing and their failure to make grammatically precise sentences can be traced back to such negative influence of these thought patterns. For instance, the error of voice in students' writing is prevalent in CLEC. It is tagged as vp7 which means error in the use of voice: active for passive or vice verse. The following table is the distribution of such errors in CLEC:

Table 4.2 The distribution of vp7 in CLEC

\begin{tabular}{|l|l|}
\hline CLEC text & Vp7 \\
\hline St3.txt & 121 \\
\hline St4.txt & 119 \\
\hline 1 million words & 593 \\
\hline
\end{tabular}

A few examples of such errors are listed below:

[1]With two children to support, his family was really [vp7 -1] faced with financial problems.

[2] All the people [ vp7 -1] struke [fm1,-] by the lovely monkeys.

[3] As time passers [fm1,-] by, he also make [ vp7 2-] ill by the polluted air. 
[4] Television can use [ vp7 s-] to explain part of the main reason that

It can be seen that the students are confused about when and how to use passive voice. They could not use it properly, and even over use it when it is actually an active sentence, such as example [1]. However, most of the mistakes are made because they tend to use active voice when it should be a passive one, such as examples [2], [3],[4]. To summarize, active voice is much more frequently used in Chinese than in English due to their differences in thought patterns.

\section{The Preference for the application of subjective words}

As is mentioned in subjective thought pattern that man is the subject of cognition, which means that man starts looking at the world from themselves. This is just the reason why the Chinese tend to think in terms of intuition. This is just against the rational and analytical thought patterns of the native English speakers in which human potentialities and creativity have been given full scope. And Judgments and theories are developed after carrying out some empirical observations and experiments on the object. Thus, they tend to prize objectivity, empirical evidence and the scientific method, which often clashes with the values of subjectivity and intuition. That is also why passive voice is so prevalent in English. Influenced by such thought patterns, the Chinese attempt to use much more subjective words to express themselves than native English speakers when writing. Evidence can be seen from the following data collected from CLEC and its contrastive corpora. The author makes a concordance about the frequency of "I think" and "I believe" occurred in these corpora. Details can be seen from the following table:

Table 4.3 The Frequency of "I think" "I believe" in CLEC

\begin{tabular}{|l|l|l|l|}
\hline subjective words & CLEC & BROWN & FOB \\
\hline I think & 1248 & 120 & 166 \\
\hline I believe & 148 & 48 & 44 \\
\hline we must & 720 & 89 & 88 \\
\hline
\end{tabular}

As can be seen that there is a tremendous contrast between CLEC and its contrastive corpora in the use of subjective words. This can further indicate that English learners tend to think much more subjectively when writing than the native English speakers. Thus, it is assumed that the preference for subjective words in students' writing is another influence of Chinese subjective thought patterns on English learning.

\section{Conclusion}

The purpose of this paper is to study the negative influence of Chinese subjective thought patterns on English learning. It is a corpus-based study with corpus as a major source of data collection. Through the process of analysis and data collection, two valuable information could be summarized: Firstly, due to the development of computer, corpus could be a very powerful, convenient and useful way in the studying of a certain language phenomenon. Secondly, it can be seen from the analysis that besides the low-proficiency level of Chinese English learners, the subjective thought pattern of Chinese could exert a negative influence on the proficient use of English in one way or another. Therefore, the key to learn English is supposed to overcome the negative influence of the Chinese thought patterns and take a good advantage of the eastern thought patterns. Hoping the paper could contribute to English learning and teaching as well as the final eradication of Chinglish in China.

\section{References}

[1] Chad Chanson. An exploration of Chinese Thought patterns [J]. Transactions of the Philological Society, 1992.

[2] Joan Pinkham. The Translator's Guide to Chinglish [M]. BeiJing: Foreign Language Teaching and Research Press. 1998

[3] Li , C. \& Thompson, S. Subject and Topic: A New Typology of Language [A]. In Li, C.(ed.). Subject and Topic [C]. New York: Academic Press, 1976. 